\title{
Serum Inflammatory Biomarkers in Patients with Nonarteritic Anterior Ischemic Optic Neuropathy
}

\author{
Nurullah Koçak ${ }^{1}$, Bilge Eraydın², Mustafa Turunç ${ }^{1}$, Volkan Yeterr, İnci Güngör \\ ${ }^{I}$ Department of Ophthalmology, Ondokuzmayls University Hospital, Samsun, Turkey \\ ${ }^{2}$ Department of Ophthalmology, Erbaa State Hospital, Tokat, Turkey
}

\begin{abstract}
Purpose: To evaluate the neutrophil-to-lymphocyte ratio (NLR), monocyte-to-lymphocyte ratio (MLR), platelet-to-lymphocyte ratio (PLR), and systemic immune-inflammation index (SII) in patients with non-arteritic anterior ischemic optic neuropathy (NAION).

Methods: Fifty-six patients with NAION and 60 age-sex matched healthy controls were included in the study. Demographic characteristics and laboratory findings of the patients and the controls were obtained from the electronic medical records. NLR, PLR, MLR, and SIl were calculated and compared between the groups. Cutoff values were also determined.

Results: Neutrophil, monocyte and platelet counts were higher in the NAION group than in the control group, but the difference was not statistically significant $(p>0.05)$. The mean NLR and SII were higher in the NAION group than in the control group ( $p=0.004$ and $p=0.011$, respectively). In the receiver operating characteristic curve analysis, the areas under the curve for NLR were 0.67 , and NLR $>1.79$ predicted NAION with a sensitivity of $71 \%$ and specificity of $59 \%$. The areas under the curve for SII was 0.66 , and SII of $>417$ predicted NAION with a sensitivity of $71 \%$ and specificity of $49 \%$. There was no significant difference in PLR and MLR between the groups ( $p=0.105$ and $p=0.347$, respectively).

Conclusions: The current study demonstrated that NAION patients had increased NLR and SII levels compared with control subjects. Elevated NLR and SII might serve as readily available inflammatory predictors in NAION patients.
\end{abstract}

Key Words: Monocyte-to-lymphocyte ratio, Neutrophil-to-lymphocyte ratio, Non-arteritic ischemic optic neuropathy, Platelet-to-lymphocyte ratio, Systemic immune-inflammation index

Non-arteritic anterior ischemic optic neuropathy (NAION) is the most common cause of optic neuropathy in patients aged $>50$ years. NAION presents with acute, painless vision loss, optic disc swelling, and visual field

Received: May 27, 2020 Final revision: October 14, 2020 Accepted: October 20, 2020

Corresponding Author: Nurullah Koçak, MD. Department of Ophthalmology, Ondokuzmayis University Hospital, Atakum 55200, Samsun, Turkey. Tel: 90-362-312-1919, Fax: 90-531-760-6820, E-mail: nurullah. kocak@omu.edu.tr defects in the affected eye. Although the pathological mechanism of NAION has not been elucidated, acute ischemia caused by circulatory insufficiency of the optic nerve head is generally accepted in the pathogenesis of NAION $[1,2]$. It is thought that multifactorial disorders, wherein different combinations of some local and systemic risk factors, play a role in the development of NAION [3]. While systemic diseases such as diabetes mellitus, hypertension, nocturnal arterial hypotension, ischemic heart disease, cerebrovascular events, and atherosclerosis are 
among the causes of circulatory failure, current studies have suggested that inflammatory markers can play an important role in the development of NAION $[4,5]$.

Recently, clinical laboratory markers of systemic inflammatory status derived from complete blood count, including the neutrophil-to-lymphocyte ratio (NLR), platelet-to-lymphocyte ratio (PLR), monocyte-to-lymphocyte ratio (MLR), and systemic immune-inflammation index (SII) have been proposed as prognostic indicators in several systemic diseases [6-10]. These markers have also been evaluated in ocular diseases such as primary angle-closure glaucoma, retinal vein occlusion, age-related macular degeneration, diabetic retinopathy, keratoconus, and pseudoexfoliation glaucoma. In addition, they have also been used as prognostic markers [11-14]. There is evidence that secondary inflammation at the site of ischemia may contribute to or produce more visual loss $[15,16]$. On the other hand, some studies argued that alterations in platelet function and microvascular blood flow are more important than the inflammatory reactions in the pathogenesis of NAION. However, the exact mechanism is still unclear [17]. These inflammatory markers may be used as predictive factors and new biomarkers for NAION. Therefore, we aimed to study whether there were any associations between NLR, PLR, MLR, and SII with NAION. To the best of our knowledge, this is the first study to evaluate the PLR, MLR, and SII in patients with NAION.

\section{Materials and Methods}

This retrospective, cross-sectional study was conducted in the Department of Ophthalmology of Ondokuz Mayis University between January 2011 and October 2018. The study protocol was approved by the Ethics Committee of Ondokuz Mayıs University (2020/666) and adhered to the tenets of the Declaration of Helsinki. A total of 56 patients diagnosed with NAION and 60 age-sex matched volunteers with similar risk factors for systemic disorders, including hypertension and diabetes mellitus, were included in the study. Control subjects were recruited from the outpatient clinic of the Department of Ophthalmology. Informed consent was obtained during the routine ophthalmological examination of control subjects who agreed to participate in the study. The diagnostic criteria for NAION were; sudden onset of painless visual loss in one eye; optic disc swelling; a relative afferent pupillary defect and a visual-field defect consistent with NAION. Subjects with any systemic diseases, including hepatic or renal failure, atrial fibrillation, chronic smoking, alcohol abuse, active systemic infection, chronic inflammatory disease or autoimmune disease, history of retinal disease, or any ocular surgery within three months, and current medical treatment that may affect blood parameters such as corticosteroids and chemotherapeutic agents were excluded. All patients underwent a comprehensive ophthalmological examination, including best-corrected visual acuity, slit-lamp biomicroscopy, intraocular pressure measurement, visual field test (Humphrey Field Analyzer; Carl Zeiss Meditec, Dublin, CA, USA), and dilated fundus examination. Baseline characteristics such as age, sex, ocular findings, systemic diseases, and drug history were recorded.

Blood sampling was performed at the time of diagnosis in the NAION group. Venous blood samples were collected after a fasting period of 12 hours from all participants for the evaluation of hematological parameters and assessed using an automated hematology analyzer (XN-3000; Sysmex America, Mundelein, IL, USA). Levels of neutrophils, lymphocytes, monocytes, platelets, red blood cells, and white blood cells were measured as part of the automated complete blood count. The NLR, PLR, and MLR were calculated as the ratio of neutrophils to lymphocytes, platelets to lymphocytes, and monocytes to lymphocytes, respectively. The SII was calculated from peripheral blood platelets, neutrophils, and lymphocytes per liter according to the equation: $\mathrm{SII}=$ platelet $\times$ neutrophil $/$ lymphocyte.

All quantitative measurements were expressed as the mean \pm standard deviation. IBM SPSS Statistics ver. 21.0 (IBM Corp., Armonk, NY, USA) was used for all statistical analyses. Student's $t$-test was used to compare qualitative data with normal distribution, and the Mann-Whitney $U$-test was used to compare parameters without normal distribution. Univariate logistic regression analysis was used to evaluate the associations between hematological parameters and NAION. A receiver operating characteristic curve (ROC) analysis was performed to determine the cutoff threshold and to quantify the accuracy of the parameters. The predictive validities were quantified as areas under the curve. A $p$-value of $<0.05$ was considered statistically significant. 


\section{Results}

Seventy subjects $(60.3 \%)$ were female, and 46 participants $(39.7 \%)$ were male. The mean age of the NAION and control groups was $64.7 \pm 10.1$ (range, 40 to 83 ) years and $63.7 \pm 12.2$ (range, 45 to 90 ) years, respectively. There were no statistical differences between the groups in terms of age, gender, and systemic disorders $(p>0.05)$. The characteristics of the participants are shown in Table 1.

Table 1. Demographic characteristics and systemic disorders within the groups

\begin{tabular}{lccc}
\hline Characteristics & $\begin{array}{c}\text { NAION } \\
(\mathrm{n}=56)\end{array}$ & $\begin{array}{c}\text { Control } \\
(\mathrm{n}=60)\end{array}$ & $p$-value \\
\hline Age (yr) & $64.7 \pm 10.1$ & $63.7 \pm 12.2$ & 0.424 \\
Male : female & $24: 37$ & $22: 33$ & 0.950 \\
Hypertension & 12 & 7 & 0.591 \\
$\begin{array}{l}\text { Diabetes mellitus } \\
\text { Hypertension and } \\
\text { diabetes mellitus }\end{array}$ & 8 & 7 & 0.736 \\
\hline
\end{tabular}

Values are presented as mean \pm standard deviation or number. $\mathrm{NAION}=$ non-arteritic anterior ischemic optic neuropathy.
Hematological parameters such as neutrophil, monocyte, and platelet counts were higher in the NAION group than in the control group, but the difference was not statistically significant. Lymphocyte levels tended to be lower in the NAION group than in the control group ( $p=0.087)$. The mean NLR and SII values were significantly higher in the NAION group than in the control group ( $p=0.004$ and $p$ $=0.011$, respectively) (Fig. 1A, 1B). However, there were no significant differences in the PLR and MLR between the groups ( $p=0.105$ and $p=0.347$, respectively). A comparison of blood parameters in the study groups is shown in Table 2. The NLR and SII were found to be significantly associated factors with NAION in univariate logistic regression analysis ( $95 \%$ confidence interval, 0.05 to $0.848 ; p$ $=0.04 ; 95 \%$ confidence interval, 17.1 to $240.9 ; p=0.02$ ).

According to the receiver operator characteristics curve analysis, the areas under the ROC curve of NLR and SII to predict patients with NAION were found to be 0.669 and 0.656 , respectively. The optimal cut-off value of NLR was $>1.79$, with $71.4 \%$ sensitivity and $58.5 \%$ specificity. The SII to predict NAION was $>417.1$ with a sensitivity of $71.4 \%$ and specificity of $48.8 \%$ (Fig. 2).
A

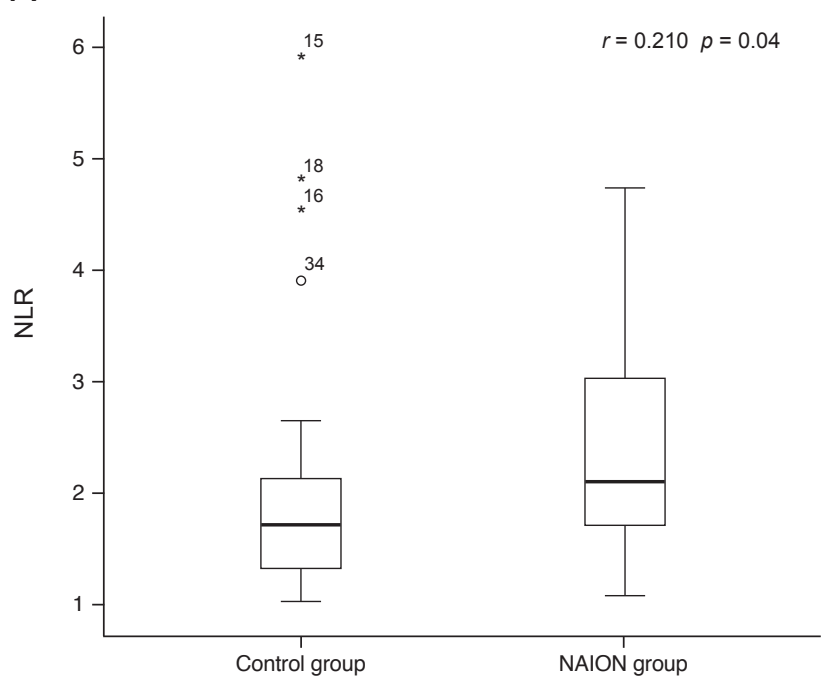

B

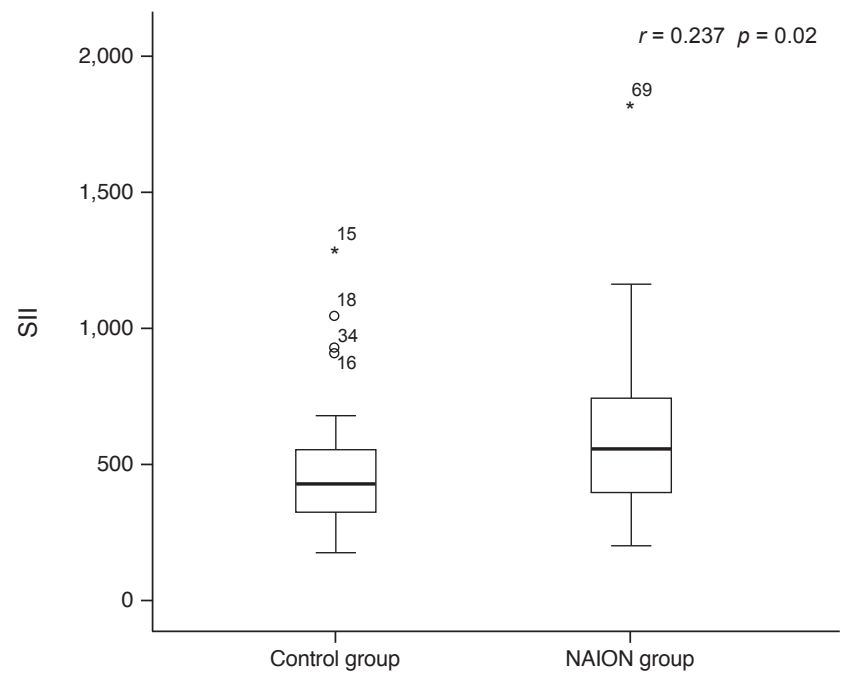

Fig. 1. Box plots demonstrating the comparison of two groups in terms of neutrophil-to-lymphocyte ratio (NLR) and systemic immune-inflammation index (SII). (A) Comparison of NLR in the control versus non-arteritic anterior ischemic optic neuropathy (NAION) groups $(p=0.004)$. (B) Comparison of SII in the control versus NAION groups $(p=0.011)$. 
Table 2. Comparison of laboratory parameters between NAION group and control group

\begin{tabular}{lccc}
\hline & NAION $(\mathrm{n}=50)$ & Control $(\mathrm{n}=44)$ & $p$-value \\
\hline $\begin{array}{c}\text { Neutrophils } \\
\left(\times 10^{9} / \mathrm{L}\right)\end{array}$ & $4.48 \pm 1.78$ & $3.86 \pm 1.14$ & 0.057 \\
$\begin{array}{c}\text { Monocytes } \\
\left(\times 10^{9} / \mathrm{L}\right)\end{array}$ & $0.49 \pm 0.21$ & $0.48 \pm 0.14$ & 0.824 \\
$\begin{array}{c}\text { Lymphocytes } \\
\left(\times 10^{9} / \mathrm{L}\right)\end{array}$ & $1.93 \pm 0.54$ & $2.16 \pm 0.64$ & 0.087 \\
$\begin{array}{l}\text { Platelets } \\
\left(\times 10^{9} / \mathrm{L}\right)\end{array}$ & $249.89 \pm 59.58$ & $243.60 \pm 60.73$ & 0.483 \\
NLR & $2.57 \pm 1.49$ & $1.98 \pm 1.05$ & 0.004 \\
MLR & $0.27 \pm 0.12$ & $0.23 \pm 0.08$ & 0.347 \\
PLR & $139.81 \pm 56.72$ & $121.28 \pm 39.89$ & 0.105 \\
SII & $600.58 \pm 293.56$ & $471.55 \pm 228.80$ & 0.011 \\
\hline
\end{tabular}

Values are presented as mean \pm standard deviation.

NAION $=$ non-arteritic anterior ischemic optic neuropathy; NLR = neutrophil-to-lymphocyte ratio; PLR = platelet-to-lymphocyte ratio; $\mathrm{MLR}=$ monocyte-to-lymphocyte ratio; $\mathrm{SII}=$ systemic immune inflammation index.

\section{Discussion}

This report demonstrates an association between serum inflammation markers and NAION. In our study, we showed that NLR and SII values were higher in the NAION group than in the control group; however, no significant difference was found in terms of MLR and PLR between the groups. To our knowledge, this is the first study to evaluate the MLR, PLR, and SII in patients with NAION.

The best evidence suggests that NAION is caused by an infarction in the region of the optic nerve head. Although the etiology of NAION is not definitively known, predisposing factors, such as local factors (small optic nerves, and disc drusen) and vascular inflammatory disease (sleep apnea syndrome, hypertension, diabetes, etc.) play a role in the development of the disease. In addition, Bernstein et al. [18] tested optic nerve changes in primate NAION models and demonstrated that early post-infarct events showed an inflammatory response and recommended that modulation of inflammation might be helpful in the management of NAION. In this perspective, we aimed to analyze potential inflammation predictors such as NLR, MLR, PLR, and SII, a relatively new marker, in NAION.

Neutrophil, lymphocyte, monocyte, and platelet counts

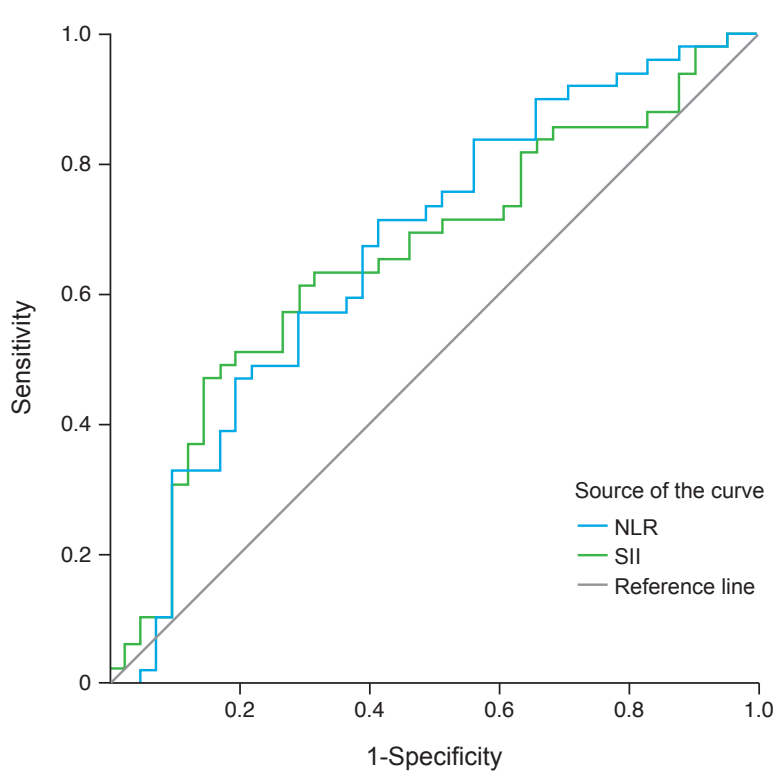

Fig. 2. Receiver operator characteristic curve showing specificity and sensitivity percentages of neutrophil-to-lymphocyte ratio (NLR) and systemic immune-inflammation index (SII) in patients with non-arteritic anterior ischemic optic neuropathy.

and ratios of these values, which have been emphasized as the predictors of inflammatory conditions in recent years, can be obtained easily from the complete blood count. NLR may be preferable because of the ratio calculated from the counts of two different immune pathway products. Neutrophils are effective in ongoing active non-specific inflammation through the secretion of various inflammatory enzymes and mediators. In contrast to the phagocytic and killing effects of neutrophils, lymphocytes regulate the immune system. It may be more predictive than either parameter alone [19]. SII is also used as a prognostic marker to evaluate the inflammatory and immune status of patients simultaneously, taking into account platelet counts and NLR (SII, platelet counts $\times$ neutrophil / lymphocyte ratio). In addition, higher SII values have been previously reported to be associated with poor outcomes in cancer patients [20]. Therefore, increased NLR and SII may represent the impaired balance between proinflammatory and anti-inflammatory status in NAION patients. Polat et al. [21] reported that patients with NAION had a higher NLR than those of the controls. In the ROC curve analysis, the cut-off value of NLR for predicting NAION was 1.94 , with $60 \%$ sensitivity and $63 \%$ specificity. Similarly, Gunes et al. [22] found considerably elevated NLR levels in NAION patients than in healthy subjects, but in 
their study, the cut-off value for NLR was 1.64 , with $85 \%$ sensitivity and $41 \%$ specificity. In both studies, NLR correlated with visual results. In patients with higher NLRs, the initial and final visual acuities were significantly poorer than in patients with lower NLRs. In the current study, we found similar results with those of previous studies that the cut-off value of NLR for predicting NAION was 1.79, with $71.4 \%$ sensitivity and $58.5 \%$.

Two different mechanisms may explain the increased NLR value in our study. First, neutrophil-mediated cellular inflammation has been shown to play a role in the early phase of NAION $[18,23]$. Some studies have demonstrated that increased neutrophil count is related to areas of ischemic injury [24-26]. The association between neutrophilia and impaired microvascular perfusion may be a manifestation of neutrophil-mediated microvascular plugging. The second possible mechanism is the association between atherosclerosis and low-grade inflammation. The pathogenesis and progression of atherosclerotic lesions are complex processes in which immune cells and various inflammatory factors play an important role [27,28]. Considering that atherosclerosis is also a risk factor for NAION, higher NLR values may indicate low-grade chronic inflammation. Similar to the NLR, higher SII values were found in NAION patients compared to controls, which have not been reported yet. Higher SII values were attributable to changes in two separate components according to the equation (SII $=$ platelet $\times$ neutrophil $/$ lymphocyte). Platelet count has been previously studied in NAION and generally was found to be higher than that in the control group $[21,29]$. We found a higher platelet count in the NAION group than in the control group, consistent with previous studies. Increased NLR values and platelet counts can cause higher SII values in the current study. According to our results, NLR and SII may help identify the risk for NAION.

The PLR provides information about both hemostasis and inflammation pathways. Activated platelets play a key role in the initiation and progression of atherosclerosis. We speculated that taking into account the effect of atherosclerosis on the pathogenesis of NAION, PLR may provide valuable information about the clinical diagnoses of NAION [30]. Recently, increased PLR values were determined in patients with primary open-angle glaucoma and retinal vein occlusion, and it was emphasized that PLR might be useful as a biomarker to predict the development and progression of these diseases [31,32]. In the current study, although the PLR was found to be higher in patients with NAION, no statistically significant difference was found.

Similar to activated platelets, an increased number of peripheral blood monocytes and macrophages increases the inflammatory reaction process, the degree of coronary stenosis, and the risk of atherosclerotic plaque rupture. High total monocyte count was associated with an increased rate of cardiovascular death, acute myocardial infarction, or non-hemorrhagic stroke in more than 950 patients referred for elective coronary angiography, as was published recently [33]. In addition to cardiovascular diseases, MLR has also been used as a marker in malignancies and inflammatory diseases [34]. However, our literature review revealed that no previous study had been carried out, that investigated the MLR ratio in patients with NAION. In our study, no difference was observed between the two groups in terms of MLR results.

This study has several limitations. First, the study had a retrospective design and included a relatively small sample size, which might have influenced the statistical significance. In addition, the small sample size may contribute to a lack of adjustment for underlying medical conditions in the statistical analysis. Second, the use of a single blood sample will not predict the persistence of blood parameters over time, and longitudinal studies would be more appropriate for evaluating predictive power.

In conclusion, although the inflammation parameters observed in the current study were higher in patients with NAION, only NLR and SII were found to be significantly different from the control group. We suggest that NLR and SII, which are easy to calculate, can easily be applied and cost-effective, may be used as readily available inflammatory predictors in NAION. The role of inflammation in the etiology of NAION will be better understood through future clinical trials and laboratory tests.

\section{Conflict of Interest}

No potential conflict of interest relevant to this article was reported. 


\section{References}

1. Hayreh SS, Joos KM, Podhajsky PA, Long CR. Systemic diseases associated with nonarteritic anterior ischemic optic neuropathy. Am J Ophthalmol 1994;118:766-80.

2. Johnson LN, Arnold AC. Incidence of nonarteritic and arteritic anterior ischemic optic neuropathy. Population-based study in the state of Missouri and Los Angeles County, California. J Neuroophthalmol 1994;14:38-44.

3. Hayreh SS. Ischemic optic neuropathies: where are we now? Graefes Arch Clin Exp Ophthalmol 2013;251:1873-84.

4. McCulley TJ, Lam BL, Feuer WJ. A comparison of risk factors for postoperative and spontaneous nonarteritic anterior ischemic optic neuropathy. $J$ Neuroophthalmol 2005;25:224.

5. Arnold AC. Pathogenesis of nonarteritic anterior ischemic optic neuropathy. J Neuroophthalmol 2003;23:157-63.

6. Feng F, Tian Y, Liu S, et al. Combination of PLR, MLR, MWR, and tumor size could significantly increase the prognostic value for gastrointestinal stromal tumors. Medicine (Baltimore) 2016;95:e3248.

7. Serfozo G, Horvath T, Fodesi I, et al. The monocyte-to-lymphocyte ratio correlates with psycho-neuro-inflammatory factors in patients with stable coronary artery disease. $\mathrm{Neu}$ roimmunomodulation 2016;23:67-74.

8. Mertoglu C, Gunay M. Neutrophil-Lymphocyte ratio and Platelet-Lymphocyte ratio as useful predictive markers of prediabetes and diabetes mellitus. Diabetes Metab Syndr 2017;11 Suppl 1:S127-31.

9. Fest J, Ruiter R, Mulder M, et al. The systemic immune-inflammation index is associated with an increased risk of incident cancer-A population-based cohort study. Int $J$ Cancer 2020;146:692-8.

10. Hu B, Yang XR, Xu Y, et al. Systemic immune-inflammation index predicts prognosis of patients after curative resection for hepatocellular carcinoma. Clin Cancer Res 2014;20:6212-22.

11. Pillunat KR, Hermann C, Spoerl E, Pillunat LE. Analyzing biomechanical parameters of the cornea with glaucoma severity in open-angle glaucoma. Graefes Arch Clin Exp Ophthalmol 2016;254:1345-51.

12. Li S, Cao W, Han J, et al. The diagnostic value of white blood cell, neutrophil, neutrophil-to-lymphocyte ratio, and lymphocyte-to-monocyte ratio in patients with primary angle closure glaucoma. Oncotarget 2017;8:68984-95.

13. Dursun A, Ozturk S, Yucel H, et al. Association of neutro- phil/lymphocyte ratio and retinal vein occlusion. Eur $J$ Ophthalmol 2015;25:343-6.

14. Ilhan N, Daglioglu MC, Ilhan O, et al. Assessment of neutrophil/lymphocyte ratio in patients with age-related macular degeneration. Ocul Immunol Inflamm 2015;23:287-90.

15. Hayreh SS. Ischemic optic neuropathy. Prog Retin Eye Res 2009;28:34-62.

16. Hayreh SS. Ischaemic optic neuropathy. Indian J Ophthalmol 2000;48:171-94.

17. Inanc $\mathrm{M}$, Tekin $\mathrm{K}$, Budakoglu $\mathrm{O}$, et al. Could platelet indices and neutrophil to lymphocyte ratio be new biomarkers for differentiation of arteritic anterior ischemic neuropathy from non-arteritic type? Neuroophthalmology 2018;42:28794.

18. Bernstein SL, Johnson MA, Miller NR. Nonarteritic anterior ischemic optic neuropathy (NAION) and its experimental models. Prog Retin Eye Res 2011;30:167-87.

19. Azab B, Zaher M, Weiserbs KF, et al. Usefulness of neutrophil to lymphocyte ratio in predicting short- and longterm mortality after non-ST-elevation myocardial infarction. Am J Cardiol 2010;106:470-6.

20. Yang R, Chang Q, Meng X, et al. Prognostic value of Systemic immune-inflammation index in cancer: a meta-analysis. J Cancer 2018;9:3295-302.

21. Polat O, Yavas GF, Inan S, Inan UU. Neutrophil-to-lymphocyte ratio as a marker in patients with non-arteritic anterior ischemic optic neuropathy. Balkan Med J 2015;32:382-7.

22. Gunes A, Yigit M, Tok L, Tok O. Neutrophil to lymphocyte ratio in patients with nonarteritic anterior ischemic optic neuropathy. Korean J Ophthalmol 2017;31:159-64.

23. Salgado C, Vilson F, Miller NR, Bernstein SL. Cellular inflammation in nonarteritic anterior ischemic optic neuropathy and its primate model. Arch Ophthalmol 2011;129:158391.

24. Pitsavos C, Tampourlou M, Panagiotakos DB, et al. Association between low-grade systemic inflammation and type 2 diabetes mellitus among men and women from the ATTICA study. Rev Diabet Stud 2007;4:98-104.

25. Nakanishi N, Sato M, Shirai K, et al. White blood cell count as a risk factor for hypertension; a study of Japanese male office workers. J Hypertens 2002;20:851-7.

26. Brooks GC, Blaha MJ, Blumenthal RS. Relation of C-reactive protein to abdominal adiposity. Am J Cardiol 2010;106: 56-61.

27. Pant S, Deshmukh A, Gurumurthy GS, et al. Inflammation and atherosclerosis: revisited. J Cardiovasc Pharmacol 
Ther 2014;19:170-8.

28. Hansen PR. Chronic inflammatory diseases and atherosclerotic cardiovascular disease: innocent bystanders or partners in crime? Curr Pharm Des 2018;24:281-90.

29. Zotz RB, Finger C, Scharf RE, Unsold R. Associations between thrombophilic risk factors and determinants of atherosclerosis and inflammation in patients with non-arteritic anterior ischaemic optic neuropathy. Hamostaseologie 2016;36:46-54.

30. Schumacher D, Strilic B, Sivaraj KK, et al. Platelet-derived nucleotides promote tumor-cell transendothelial migration and metastasis via P2Y2 receptor. Cancer Cell 2013;24:1307.

31. Ozgonul C, Sertoglu E, Mumcuoglu T, Kucukevcilioglu M.
Neutrophil-to-lymphocyte ratio and platelet-to-lymphocyte ratio as novel biomarkers of primary open-angle glaucoma. J Glaucoma 2016;25:e815-20.

32. Sahin M, Elbey B, Sahin A, et al. Neutrophil-to-lymphocyte ratio and platelet-to-lymphocyte ratio in retinal vein occlusion. Clin Exp Optom 2020;103:490-4.

33. Rogacev KS, Cremers B, Zawada AM, et al. CD14++CD16+ monocytes independently predict cardiovascular events: a cohort study of 951 patients referred for elective coronary angiography. J Am Coll Cardiol 2012;60:1512-20.

34. Du J, Chen S, Shi J, et al. The association between the lymphocyte-monocyte ratio and disease activity in rheumatoid arthritis. Clin Rheumatol 2017;36:2689-95. 